\title{
The Fast Screening Method of Biological Samples for Early Diagnosis of Gastric Cancer
}

\author{
Raluca-Ioana Stefan-van Staden ${ }^{1, ~ *, ~ R u x a n d r a-M a r i a ~ I l i e-M i h a i ~}{ }^{1}$, Simona \\ Gurzu $^{2,3,4}$ \\ ${ }^{1}$ Laboratory of Electrochemistry and PATLAB, National Institute of Research \\ for Electrochemistry and Condensed Matter, Bucharest, Romania \\ ${ }^{2}$ Department of Pathology, George Emil Palade University of Medicine, \\ Pharmacy, Sciences and Technology, Targu-Mures, Romania \\ 3 Department of Pathology, Clinical County Emergency Hospital, Tar- \\ gu-Mures, Romania \\ ${ }^{4}$ Department of Pathology, Research Center (CCAMF), Targu-Mures, Romania \\ *Corresponding author: Prof. Dr. Habil. Raluca-Ioana Stefan-van Staden, \\ Laboratory of Electrochemistry and PATLAB, National Institute of Research \\ for Electrochemistry and Condensed Matteer, 202, Splaiul Independentei Str., \\ 060021, Bucharest, Romania. Tel: +40751507779; Fax: +40213163113; \\ E-mail: ralucavanstaden@gmail.com
}

DOI: $10.30699 /$ mci.4.3.25

Submitted: 13 May 2020

Revised: 1 June 2020

Accepted: 17 June 2020

e-Published: 1 July 2020

\section{Keywords:}

Stomach Neoplasms

Early Diagnosis

Early Detection of Cancer
Introduction: Early diagnosis of gastric cancer is the best solution for reversing cancer and curing patients. So far, gastric cancer is diagnosed in late stages due to misinterpretation of symptoms or absence of specific symptoms. Highly sensitive and selective detection methods for biomarkers are needed for early detection.

Methods: This paper proposed a new fast screening method, based on the utilization of a new stochastic microsensor, able to reliably perform the molecular recognition and quantitative determination of three biomarkers including CA19-9, CEA, and p53 in biological samples such as whole blood, saliva, urine, and tumoral tissue.

Results: The limits of determination were lower than those used for the standard assay of these biomarkers in the specialized laboratories (e.g., ELISA, chemiluminescence) and this can facilitate the determination of CA19-9, CEA, and p53 at very low levels in biological fluids. The validation of the method was done; using biological samples (urine, saliva, whole blood, and tumoral tissues) from confirmed patients.

Conclusions: The proposed tools and method proposed in this paper can be used as a screening test for early diagnosis of gastric cancer as well as to follow up on the efficiency of the proposed treatment.

(C) 2020. Multidisciplinary Cancer Investigation

\section{INTRODUCTION}

Early diagnosis of gastric cancer is very important because only at this stage the overall survival rate can be improved. Fast and minimally invasive or noninvasive screening tests can contribute to the early detection of biomarkers in biological samples.
To date, the standard methods used for the detection of biomarkers (e.g., Carcinoembryonic antigen CEA, Carbohydrate antigen 19-9 - CA19-9, and p53) and diagnosis of gastric cancer are mainly the enzyme-linked immunosorbent assay (ELISA) 
and chemiluminescence. The main limits of these techniques consist of the necessity of using whole blood samples and significantly increased limits in advanced rather than early-stage carcinomas. Requests are also for the determination of these biomarkers in urine and saliva so that noninvasive methods may be applied especially for children $[1,2]$. There is also a need for fast detection and quantification of biomarkers in tissue samples to identify the molecular type of tumor and to be able to decide while performing surgery or preoperative chemotherapy. CEA is brought up in various malignancies such as gastric, colorectal, pancreatic, or hepatocellular carcinomas. CA19-9 is a glycoprotein highly associated with malignant tumors and commonly used as a clinical marker for the diagnosis of pancreatic, colorectal, and gastric carcinomas $[3,4]$. p53 is a nuclear phosphoprotein; considered as a tumor suppressor gene that plays a central role in the arrest, repair, and apoptosis of the cell cycle [5]. The overexpression of p53 protein can be interpreted as an indication of a high rate of cell proliferation with malignant potentials. A few examinations showed that overexpression of p53 in tumor tissue is reflected in raised degrees of $\mathrm{p} 53$ protein in serum and urine [6]. Until this point in time, numerous investigations have been committed to assessing the convenience of p53 as a marker in patients with malignancies, as anticipation devices, for observing the treatment viability and for foreseeing repetitive tumors before they are clinically distinguishable [7].

Stochastic sensors are well known for their ability to perform qualitative and quantitative analysis, as well as multianalyte assay (more than one biomarker can be determined; using the same stochastic sensor). There are some benefits to the stochastic method over ELISA and chemiluminescence methods [8, 9]. High sensitivity and selectivity, very low limits of biomarkers determination found in a complex matrix, multianalyte detection using one stochastic microsensor, low-cost system output, no need of skilled technicians, not treating the samples before the measurement, and the possibility of electrode surface refreshment are among these superiorities. However, three different kits are required to determine CA19-9, CEA, and p53 while using ELISA or chemiluminescence. Therefore, a stochastic microsensor based on nanographene modified with $\beta$-cyclodextrin $(\beta$ CD) was proposed for molecular recognition and quantification of CA19-9, CEA, and p53 in biological samples (whole blood, saliva, urine, and tumoral tissue samples) in one run taking place in $20 \mathrm{~min}$. The selection of $\beta$-cyclodextrin for the design of the stochastic sensor was done due to its structure [10-13]. It is favorable for obtaining the signal needed for stochastic sensors because the molecule could allow biomarkers inside its cavity. Graphene materials were previously used for the design of sensors. The graphene oxide was reduced in its pure [14] or it was modified [15] after the addition of $\beta$-cyclodextrin. Nanographenes were also modified with a Ru complex and $\beta$-cyclodextrin for electrochemiluminescence detection [15]. Cyclodextrin functionalized graphene nanosheets were designed for high supramolecular recognition capability in electrochemical methods [16]. This paper proposed a physical immobilization of $\beta$-cyclodextrin [17] in a nanographene paste for the design of a stochastic sensor - for which the nanographene (the support matrix) assures the stability of $\beta$-cyclodextrin which is actively involved in the generation of the specific signal for stochastic sensors.

\section{METHODS}

\section{Design of Stochastic Microsensors}

The nanographene powder was mixed with paraffin oil to form a homogeneous paste and a solution of $\beta$-cyclodextrin $(\beta-\mathrm{CD})\left(10^{-3} \mathrm{~mol} \mathrm{~L}^{-1}\right)$ was added to the paste. The obtained paste was introduced in a plastic tube (obtained in our laboratory; using the $3 \mathrm{D}$ printer) with the diameter of the internal active surface of $120 \mu \mathrm{m}$ (the diameter was programmed; using the computer and checked; using the scanning electron microscope (SEM)). A silver wire was inserted in the tube to serve as electric contact. Between measurements, the sensor was washed with deionized water and dried with paper. If not used, the sensor was kept in a dry place at room temperature. The characterization of the modified nanographene paste was done; using the Atomic force microscopy (AFM) (Figure 1).

\section{Description of the Screening Test}

All measurements were performed with an AUTOLAB/PGSTAT 12 (Metrohm) connected 


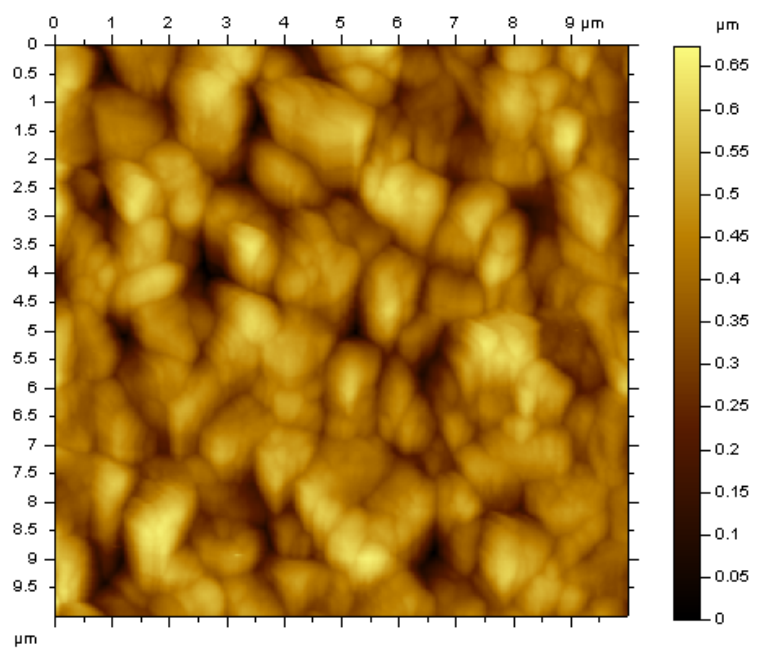

Figure 1: Atomic Force Microscopy (AFM) Topographical 2D Image for the Surfaces of the Sensor

to a computer. The electrochemical cell employed comprised the stochastic microsensor, $\mathrm{Ag} / \mathrm{AgCl}$ $\left(0.1 \mathrm{~mol} \mathrm{~L}^{-1} \mathrm{KCl}\right)$ reference electrode, and a Pt wire as the auxiliary electrode. The stochastic model was used for the measurements of the qualitative

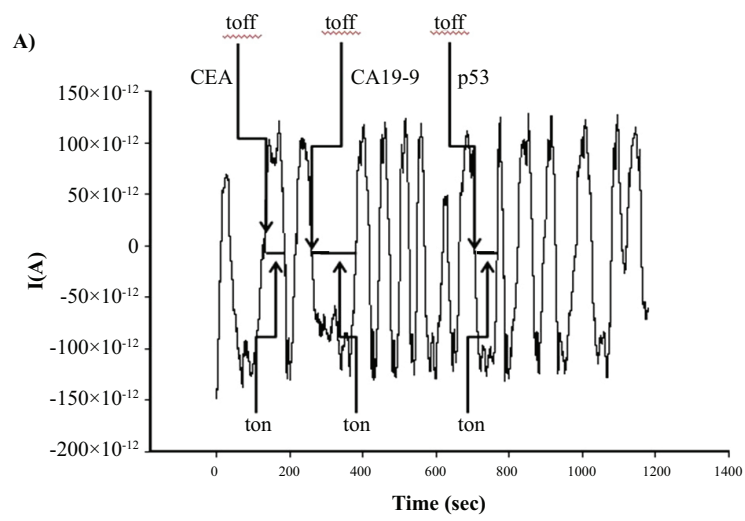

C)

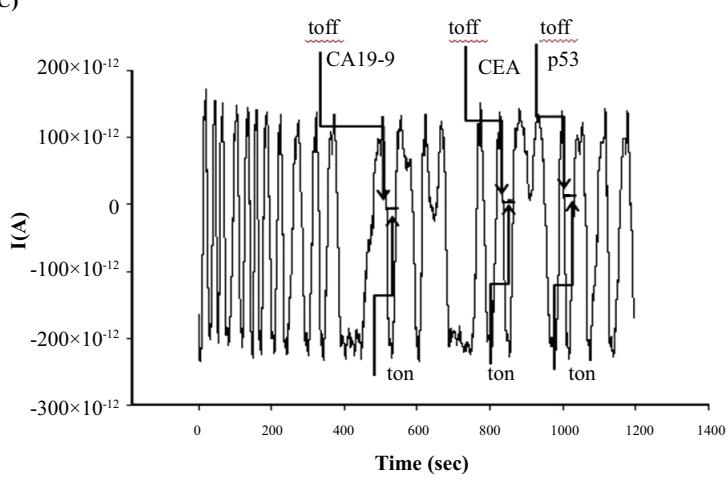

parameter $\mathrm{t}_{\text {off }}$ and the quantitative parameter $\mathrm{t}_{\text {on }}$, at a constant potential (125 mV vs. $\mathrm{Ag} / \mathrm{AgCl})$, and was used for both qualitative and quantitative analysis of p53, CA19-9, and CEA (Figure 2). The $t_{\text {off }}$ value was identified in each diagram to identify p53, CA19-9, and CEA. For their quantification, the $t_{\text {on }}$ value was read and used. The equation of calibration $\left(1 / \mathrm{t}_{\text {on }}=\mathrm{a}+\mathrm{bxConc}_{\text {biomarker }}\right)$ was obtained; using the linear regression method.

Biological samples: whole blood, saliva, urine, and tumoral tissue samples were obtained from confirmed patients with gastric cancer. Informed consent was obtained from all patients, before surgery.

\section{RESULTS}

The response of stochastic microsensors relies on the conductivity of the channel. When the biomarker is going inside the channel under an applied potential, the molecule of the biomarker is blocking it and the intensity of the current is dropping to zero for a certain time called $t_{\text {off }}$ - the signature of the biomarker - which is the stage of

B)

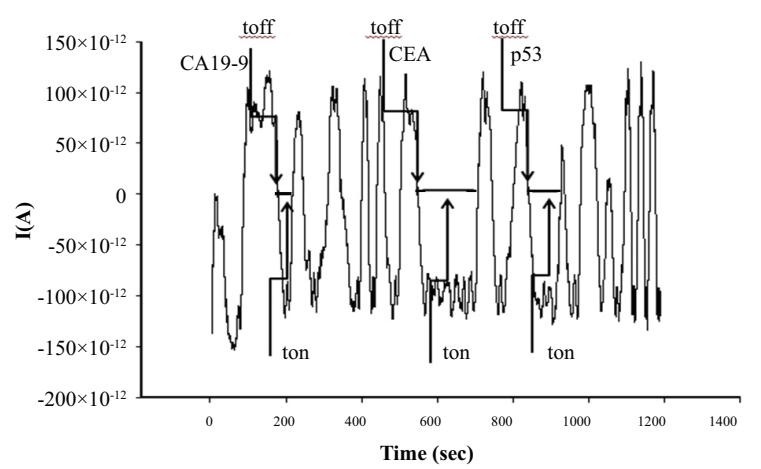

D)

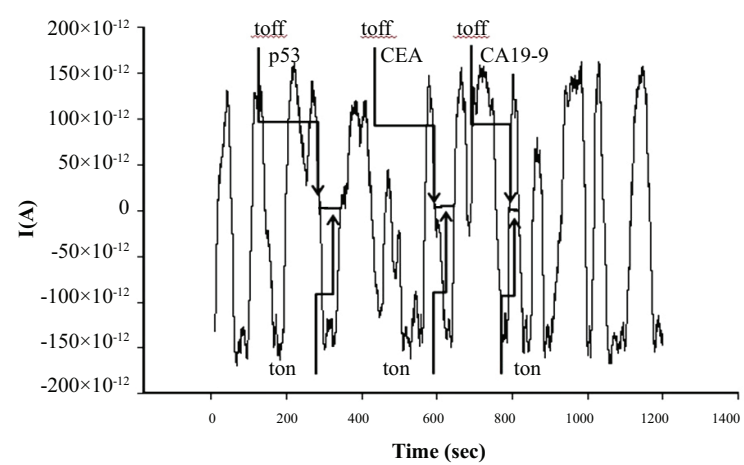

Figure 1: Pattern Recognition of CA19-9, CEA, and p53 Using the Stochastic Microsensor A) Whole blood samples; B) Saliva; C) Urine; D) Tumoral tissue samples 
molecular recognition. In the second stage - the quantification stage - called $t_{\text {on }}$ which is dependent on the concentration of the analyte, the biomarker undergoes binding and redox processes. Table 1 shows the response characteristics of the stochastic microsensor. The values recorded for the signatures $\left(\mathrm{t}_{\text {off }}\right.$ values $)$ of CEA, CA19-9, and p53 indicates that all three biomarkers can be recognized by the stochastic microsensors. The sensitivities are high and the limits of determination are lower than those indicated for ELISA or chemiluminescence methods. Also, the linear concentration range is larger than those of ELISA and chemiluminescence, making it possible to utilize the screening test from early to later stages of gastric cancer as well as following up the efficiency of the treatment.

Table 1: Response Characteristics of the Stochastic Microsensors Used for the Assay of CA19-9, CEA, and p53

\begin{tabular}{|c|c|c|c|c|c|}
\hline Biomarker & $\begin{array}{c}\text { Calibration Equation; } \\
\text { Correlation Coefficient (r) }\end{array}$ & $\begin{array}{c}\text { Linear Concentration } \\
\text { Range }\end{array}$ & $t_{\text {off }}(s)$ & $\begin{array}{l}\text { Sensitivity } \\
\left(\mathrm{s}^{-1} / \mathrm{g} \times \mathrm{mL}^{-1}\right)\end{array}$ & $\begin{array}{c}\text { Limit of } \\
\text { Determination }\end{array}$ \\
\hline CEA, g/mL & $1 / \mathrm{t}_{\text {on }}=0.04+60.80 \mathrm{C} ; \mathrm{r}=0.9998$ & $2.5 \times 10^{-12}-3.2 \times 10^{-10}$ & 1.2 & 60.80 & $2.5 \times 10^{-12}$ \\
\hline CA 19-9, U/mL & $1 / t_{\text {on }}=0.02+6.23 \times 10^{4} \mathrm{C} ; \mathrm{r}=0.9993$ & $1.6 \times 10^{-8}-1 \times 10^{2}$ & 2.4 & $6.23 \times 10^{4}$ & $1.6 \times 10^{-8}$ \\
\hline $\mathrm{p53}, \mathrm{g} / \mathrm{mL}$ & $1 / \mathrm{t}_{\text {on }}=0.03+4.22 \times 10^{-1} \mathrm{C} ; \mathrm{r}=0.9984$ & $1.6 \times 10^{-10}-4.0 \times 10^{-5}$ & 1.6 & $4.22 \times 10^{-1}$ & $1.6 \times 10^{-10}$ \\
\hline
\end{tabular}

Table 2: Quantitative Determination of CEA, CA19-9, and p53 in Whole Blood Samples ( $\mathrm{N}=10)$

\begin{tabular}{|c|c|c|c|}
\hline Sample no. & $\begin{array}{c}\text { CEA } \\
\left(n g \mathbf{~ m L}^{-1}\right)\end{array}$ & $\begin{array}{c}\text { CA19-9 } \\
\left(\mathrm{U} \mathrm{mL}^{-1}\right)\end{array}$ & $\begin{array}{c}\text { p53 } \\
\left(\text { (ng } \mathbf{~ L L}^{-1}\right)\end{array}$ \\
\hline 1 & $15.56 \pm 0.03$ & $75.63 \pm 0.05$ & $5.16 \pm 0.02$ \\
\hline 2 & $36.72 \pm 0.06$ & $621.87 \pm 0.05$ & $4.40 \pm 0.03$ \\
\hline 3 & $28.11 \pm 0.04$ & $37.80 \pm 0.03$ & $9.43 \pm 0.03$ \\
\hline 4 & $18.17 \pm 0.03$ & $57.44 \pm 0.03$ & $7.88 \pm 0.02$ \\
\hline 5 & $15.99 \pm 0.03$ & $226.83 \pm 0.04$ & $2.14 \pm 0.03$ \\
\hline 6 & $45.67 \pm 0.04$ & $172.08 \pm 0.05$ & $4.40 \pm 0.03$ \\
\hline 7 & $18.40 \pm 0.04$ & $43.12 \pm 0.05$ & $7.39 \pm 0.02$ \\
\hline 8 & $13.93 \pm 0.03$ & $44.04 \pm 0.04$ & $6.32 \pm 0.02$ \\
\hline 9 & $7.12 \pm 0.03$ & $37.39 \pm 0.04$ & $6.89 \pm 0.02$ \\
\hline 10 & $11.68 \pm 0.03$ & $53.30 \pm 0.05$ & $6.21 \pm 0.02$ \\
\hline 11 & $18.04 \pm 0.03$ & $57.79 \pm 0.03$ & $4.01 \pm 0.02$ \\
\hline 12 & $13.02 \pm 0.01$ & $73.05 \pm 0.03$ & $4.22 \pm 0.02$ \\
\hline
\end{tabular}

Table 4: Quantitative Determination of CEA, CA19-9, and p53 in Urine Samples $(\mathrm{N}=10)$

\begin{tabular}{|c|c|c|c|}
\hline Sample no. & 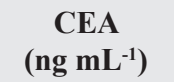 & $\begin{array}{c}\text { CA19-9 } \\
\left(\mathbf{U ~ m L}^{-1}\right)\end{array}$ & $\begin{array}{c}\text { p53 } \\
\left(\operatorname{ng~mL}^{-1}\right)\end{array}$ \\
\hline 1 & $32.20 \pm 0.05$ & $84.14 \pm 0.03$ & $8.75 \pm 0.02$ \\
\hline 2 & $36.72 \pm 0.06$ & $669.41 \pm 0.06$ & $6.83 \pm 0.04$ \\
\hline 3 & $38.63 \pm 0.04$ & $39.86 \pm 0.03$ & $8.89 \pm 0.02$ \\
\hline 4 & $36.71 \pm 0.02$ & $133.48 \pm 0.03$ & $8.90 \pm 0.02$ \\
\hline 5 & $25.94 \pm 0.04$ & $314.20 \pm 0.04$ & $4.37 \pm 0.02$ \\
\hline 6 & $83.05 \pm 0.03$ & $193.81 \pm 0.07$ & $5.88 \pm 0.02$ \\
\hline 7 & $21.45 \pm 0.03$ & $207.32 \pm 0.08$ & $6.32 \pm 0.02$ \\
\hline 8 & $22.77 \pm 0.04$ & $50.76 \pm 0.03$ & $7.26 \pm 0.01$ \\
\hline 9 & $5.40 \pm 0.05$ & $38.62 \pm 0.02$ & $7.02 \pm 0.01$ \\
\hline 10 & $11.34 \pm 0.02$ & $58.96 \pm 0.03$ & $6.89 \pm 0.02$ \\
\hline 11 & $21.45 \pm 0.04$ & $72.11 \pm 0.05$ & $8.09 \pm 0.01$ \\
\hline 12 & $23.48 \pm 0.02$ & $89.48 \pm 0.06$ & $6.22 \pm 0.01$ \\
\hline
\end{tabular}

Table 3: Quantitative Determination of CEA, CA19-9, and p53 in Saliva Samples $(\mathrm{N}=10)$

\begin{tabular}{|c|c|c|c|}
\hline Sample no. & $\begin{array}{c}\text { CEA } \\
\left(n g \mathbf{~ m L}^{-1}\right)\end{array}$ & $\begin{array}{l}\text { CA19-9 } \\
\left(\mathbf{U}^{-1} L^{-1}\right)\end{array}$ & $\begin{array}{c}\text { p53 } \\
\left(\mathrm{ng} \mathrm{mL}^{-1}\right)\end{array}$ \\
\hline 1 & $124.65 \pm 0.05$ & $123.21 \pm 0.03$ & $2.20 \pm 0.02$ \\
\hline 2 & $49.03 \pm 0.02$ & $75.80 \pm 0.03$ & $14.33 \pm 0.04$ \\
\hline 3 & $102.03 \pm 0.03$ & $1.53 \pm 0.02$ & $11.58 \pm 0.04$ \\
\hline 4 & $120.30 \pm 0.04$ & $107.03 \pm 0.05$ & $2.35 \pm 0.02$ \\
\hline 5 & $155.34 \pm 0.02$ & $157.79 \pm 0.04$ & $1.26 \pm 0.01$ \\
\hline 6 & $45.67 \pm 0.03$ & $41.26 \pm 0.03$ & $2.96 \pm 0.01$ \\
\hline 7 & $218.03 \pm 0.03$ & $19.03 \pm 0.04$ & $4.45 \pm 0.02$ \\
\hline 8 & $54.97 \pm 0.04$ & $24.76 \pm 0.03$ & $7.27 \pm 0.02$ \\
\hline 9 & $27.46 \pm 0.04$ & $11.20 \pm 0.03$ & $2.51 \pm 0.02$ \\
\hline 10 & $46.64 \pm 0.03$ & $15.62 \pm 0.02$ & $1.21 \pm 0.02$ \\
\hline 11 & $33.40 \pm 0.03$ & $247.84 \pm 0.07$ & $6.65 \pm 0.04$ \\
\hline 12 & $38.40 \pm 0.01$ & $43.43 \pm 0.05$ & $3.15 \pm 0.04$ \\
\hline
\end{tabular}

Table 5: Quantitative Determination of CEA, CA19-9, and p53 in Tumoral Tissue Samples $(\mathrm{N}=10)$

\begin{tabular}{|c|c|c|c|}
\hline Sample no. & $\begin{array}{c}\text { CEA } \\
\left(n g \mathrm{~mL}^{-1}\right)\end{array}$ & $\begin{array}{l}\text { CA19-9 } \\
\left(\mathrm{U} \mathrm{mL}^{-1}\right)\end{array}$ & $\begin{array}{c}\text { p53 } \\
\left(\mathrm{ng} \mathrm{mL}^{-1}\right)\end{array}$ \\
\hline 1 & $36.19 \pm 0.02$ & $170.95 \pm 0.02$ & $8.69 \pm 0.01$ \\
\hline
\end{tabular}

Table 6: Recovery Tests of CEA, CA19-9, and p53 in Biological Samples $(\mathrm{N}=10)$

\begin{tabular}{lccc}
\hline & CEA, \% & CA19-9, \% & p53, \% \\
\hline $\begin{array}{l}\text { Whole } \\
\text { Blood }\end{array}$ & $98.87 \pm 0.05$ & $98.14 \pm 0.07$ & $98.69 \pm 0.06$ \\
Saliva & $99.07 \pm 0.04$ & $99.95 \pm 0.03$ & $99.98 \pm 0.03$ \\
Urine & $97.45 \pm 0.07$ & $97.78 \pm 0.08$ & $98.02 \pm 0.04$ \\
$\begin{array}{l}\text { Tumoral } \\
\text { Tissue }\end{array}$ & $96.47 \pm 0.02$ & $95.98 \pm 0.03$ & $96.00 \pm 0.04$ \\
\hline
\end{tabular}


Biological samples: whole blood $(10 \mu \mathrm{L})$, saliva $(10 \mu \mathrm{L})$, urine $(10 \mu \mathrm{L})$, and tumoral tissue $(0.2 \times 0.2 \mathrm{~cm})$ samples were obtained from 12 confirmed patients with gastric cancer. Blood, saliva, and urine were collected at least two hours before surgery. All of the patients underwent surgery. In all of the cases, the fresh tumor tissue was obtained from gastrectomy specimens without affecting the histopathological assessment. The samples were screened; using the stochastic microsensor, without treating the samples before the measurements. Typical diagrams obtained for the screening of whole blood, saliva, urine, and tumoral tissue samples are shown in Figure 2. To find which of the peaks corresponds to CEA, CA19-9, or $\mathrm{p} 53$, one should check the signature of the biomarker (the value of $t_{\text {off }}$, see Table 1). After identifying the signature, the $t_{\text {on }}$ value is read and used for the determination of concentration accordingly to the stochastic model described above (Tables 2-5).

Because we could not always use the ELISA (the standard method) for the determination of the biomarkers in whole blood, saliva, urine, and tissue samples, we performed a validation/recovery test for the screening method. Therefore, in each type of sample, different amounts of CEA, CA19-9, and p53 were added (these biomarkers were determined before and after addition of the known amounts of each of them) and the recovered amount was compared with the added amount of each biomarker. The results are shown in Table 6 .

\section{DISCUSSIONS}

The results obtained from the screening of whole blood, saliva, urine, and tumoral tissue samples; using the stochastic microsensor were in the specific ranges of gastric cancer. Accordingly, the proposed screening test could be used for the fast screening test of biological samples such as whole blood, saliva, urine, and tumoral tissue samples. The ELISA method is the most used standard method in clinical laboratories. Approximately, $90 \mathrm{~min}$ is needed for analyzing each of these markers with distinct analysis kits. The linear concentration ranges are 0.34-250ng/mL for CEA, $0.30-200 \mathrm{U} / \mathrm{mL}$ for CA199 , and $0.78-50 \mathrm{U} / \mathrm{mL}$ for $\mathrm{p} 53$ (less wide than those obtained for the proposed sensor). Determination limits are as follows: $0.34 \mathrm{ng} / \mathrm{mL}$ for CEA, $0.30 \mathrm{U} /$ $\mathrm{mL}$ for CA19-9, and $0.78 \mathrm{U} / \mathrm{mL}$ for $\mathrm{p} 53$ which are far higher than those recorded using the proposed stochastic sensor. The advantage of this method over previously applied techniques is that the limits of CEA and p53 determination are lower than those using $\mathrm{N}$-modified graphene and protoporphyrin IX based stochastic sensor [15]. The results shown in Table 6 revealed that the recoveries were higher than $95.00 \%$ and the relative standard deviations were lower than $1.00 \%$. These findings proved that the screening method is highly reliable when used for screening tests of whole blood, saliva, urine, and tumoral tissues. The total analysis time for the three biomarkers; using the proposed stochastic sensor in the whole blood, urine, saliva, and tissue sample was $20 \mathrm{~min}$.

The proposed screening method is fast and reliable and could be used for the screening of different biological samples: whole blood, saliva, urine, and fresh tumoral tissue samples for CEA, CA19-9, and p53. The large linear concentration range allows its utilization for the screening of biological samples from patients found in early to later stages; making possible the early diagnosis of gastric cancer and also the monitorization of the efficiency of the treatment of gastric cancer. The feature of the screening method is its utilization for mass screening of the population to facilitate early diagnosis of gastric cancer.

\section{ACKNOWLEDGMENTS}

This work was supported by a grant of the Romanian National Authority for Scientific Research, CNCS- UEFISCDI; project number PN-III-P4-IDPCCF-2016-0006.

\section{CONFLICT OF INTEREST}

The authors declared no conflict of interest.

\section{ETHICS APPROVAL}

The study was approved by the Institutional Review Board of Istanbul University. Ethics committee approval no. 32647/2018 awarded by the Clinical County Emergency Hospital from Targu-Mures, Romania.

\section{REFERENCES}

1. Abbas M, Habib M, Naveed M, Karthik K, Dhama K, Shi M, et al. The relevance of gastric cancer biomarkers in prognosis and pre- and post- chemotherapy in clinical practice. Biomed Pharmacother. 2017;95:1082-90. DOI: 10.1016/j.biopha.2017.09.032 PMID: 28922727.

2. Yamashita K, Watanabe M. Clinical significance of tumor markers and an emerging perspective on colorectal cancer. Cancer Sci. 2009;100(2):195-9. DOI: 10.1111/j.1349- 
7006.2008.01022.x PMID: 19200256.

3. Huang Z, Liu F. Diagnostic value of serum carbohydrate antigen 19-9 in pancreatic cancer: a meta-analysis. Tumour Biol. 2014;35(8):7459-65. DOI: 10.1007/s13277-0141995-9 PMID: 24789274.

4. Duraker N, Hot S, Polat Y, Höbek A, Gençler N, Urhan N. CEA, CA 19-9, and CA 125 in the differential diagnosis of benign and malignant pancreatic diseases with or without jaundice. J Surg Oncol. 2007;95(2):142-7. DOI: 10.1002/ jso.20604 PMID: 17262731.

5. Luch A. The carcinogenic effects of polycyclic aromatic hydrocarbons. Singapore: World Scientific Publishing Co Pte Ltd; 2005. 516 p. DOI: 10.1142/p306.

6. Darabi M, Tayebi Meibodi N, Mahdavi R, Arab D. p53 protein in serum and urine samples of patients with bladder transitional cell carcinoma and its overexpression in tumoral tissue. Urol J. 2006;3(4):216-9. PMID: 17559044.

7. Zare M, Shahtaheri SJ, Mehdipur P, Shekari M, Zare S. Levels of p53 protein as biomarker in plasma of workers exposed to carcinogenic polycyclic aromatic hydrocarbons. Toxicol Environ Chem. 2013;95(1):187-96. DOI: 10.1080/02772248.2012.752489.

8. Stefan-van Staden R-I, Popa-Tudor I. Molecular recognition of C-reactive protein, adiponectin and $\mathrm{Zn} 2+$ in serum samples. J Electrochem Soc. 2019;166(9):B3051-B5. DOI: $\underline{10.1149 / 2.0111909 j \text { jes. }}$

9. Stefan-van Staden R-I, Moldoveanu I. Stochastic microsensors based on nanostructured materials used in the screening of whole blood for hepatitis B. J Electrochem Soc. 2013;161(2):B3001-B5. DOI: 10.1149/2.026312jes.

10. Wei Y, Kong L-T, Yang R, Wang L, Liu J-H, Huang X-J. Electrochemical impedance determination of polychlorinated biphenyl using a pyrenecyclodextrin-decorated single-walled carbon nanotube hybrid. Chem Commun (Camb). 2011;47(18):5340-2. DOI: 10.1039/c1cc11267h PMID: 21451857.

11. Liu Z, Zhang A, Guo Y, Dong C. Electrochemical sensor for ultrasensitive determination of isoquercitrin and baicalin based on DM- $\beta$-cyclodextrin functionalized graphene nanosheets. Biosens Bioelectron. 2014;58:242-8. DOI: 10.1016/j.bios.2014.02.051 PMID: 24657644.

12. Zhang Z, Gu S, Ding Y, Shen M, Jiang L. Mild and novel electrochemical preparation of $\beta$-cyclodextrin/graphene nanocomposite film for super-sensitive sensing of quercetin. Biosens Bioelectron. 2014;57:239-44. DOI: 10.1016/j. bios.2014.02.014 PMID: 24594590.

13. Zhu G, Zhang X, Gai P, Zhang X, Chen J. $\beta$-Cyclodextrin non-covalently functionalized single-walled carbon nanotubes bridged by 3,4,9,10-perylene tetracarboxylic acid for ultrasensitive electrochemical sensing of 9-anthracenecarboxylic acid. Nanoscale. 2012;4(18):5703-9. DOI: 10.1039/c2nr31378b PMID: 22886354.

14. Pourjavadi A, Eskandari M, Hosseini SH, Nazari M. Synthesis of water dispersible reduced graphene oxide via supramolecular complexation with modified $\beta$-cyclodextrin. Int J Polymeric Mater and Polymeric Biomater. 2017;66(5):23542. DOI: $10.1080 / 00914037.2016 .1201766$.

15. Stefan-van Staden R-I, Ilie-Mihai R-M, Pogacean F, Pruneanu S. Graphene-based stochastic sensors for pattern recognition of gastric cancer biomarkers in biological fluids. J Porphyr Phthalocyanines. 2019;23(11n12):1365-70. DOI: $10.1142 / \mathrm{s} 1088424619501293$.

16. Chen $\mathrm{C}$, Wei G, Yao X, Liao F, Peng H, Zhang J, et al. $\mathrm{Ru}$ (bpy)32+//-cyclodextrin-Au nanoparticles/nanographene functionalized nanocomposites-based thrombin electrochemiluminescence aptasensor. J Solid State Electrochem. 2018;22(7):2059-66. DOI: 10.1007/s10008-0183910-6.

17. Guo Y, Guo S, Ren J, Zhai Y, Dong S, Wang E. Cyclodextrin functionalized graphene nanosheets with high supramolecular recognition capability: synthesis and hostguest inclusion for enhanced electrochemical performance. ACS Nano. 2010;4(7):4001-10. DOI: 10.1021/nn100939n PMID: 20583782. 